\title{
THE IMPACT OF TECHNOLOGY ON RECRUITMENT PROCESS
}

\author{
Chaza Abdul, Harrisburg University of Science and Technology, cabdul@harrisburgu.edu \\ Wenli Wang, Robert Morris University, wangw@rmu.edu \\ Yating Li, Harrisburg University of Science of Technology, yli11@my.harrisburgu.edu
}

\begin{abstract}
Recruitment technologies have become widely applied by human resource (HR) professionals. What are the perceived impact of technology on the recruitment process? This research surveyed HR professionals across industries to understand their perceptions on how technology has changed their recruiting process. Results show that: 1) recruitment technology is widely used despite varied popularities; 2) recruitment technology has improved the recruitment process; 3) technology has played a more significant role at the earlier stages of recruitment processes such as sourcing and screening candidates than the later stages such as interviewing and engaging candidates; 4) it is easy to master the recruitment technology; and 5) artificial intelligence has already started changing the recruitment process. The perceived positive impact of technology is only sprouting as artificial intelligence and blockchain can further improve the recruitment process in the near future.
\end{abstract}

Keywords: Recruitment Technology, Human Resources Management, Recruitment Process, Artificial Intelligence

\section{INTRODUCTION}

Recruitment is one of the essential function of human resource management. Recruitment was simpler in old days -people worked as a blacksmith, a peasant, or a doctor throughout their lives. As time went on, people became more diverse, knowledgeable, and skillful. They started to move on and change their occupations. Since then, recruitment has become more complicated for both job seekers and job recruiters. According to the U.S. Bureau of Labor Statistics, the number of job openings was at 6.4 million on the last business day of December 2019 (BLS, 2020). At the same time, the number of hires was little changed at 5.9 million with the hires rate at 3.9 percent. The labor market was still competitive by the end of 2019. The influx of information is one of the most important factors causing the difference. A study from Glassdoor.com, an employment website, shows that every corporate job opening attracts 250 resumes on average; but only 4 to 6 of these people will be called for an interview, and only one of those will be offered a job. Employers also have struggles in hiring; the reasons for these struggles boil down to mismatched talent and wages. Using technology on recruiting could significantly simplify the screening process, reduce turnaround time, and improve the overall effectiveness of hiring process. This study aims to examine if technology does have a positive impact on recruiting.

Nearly everyone who has been a candidate for a job goes through the hiring process. Recruiters on the hiring end need to deal with information of many candidates just for one job, not the mention there are multiple job openings for just one company. With more information shared online, recruiters search for and identify the right candidates is analogous to find the needles in the haystacks. The traditional recruiting process has changed dramatically recently by leveraging technology, such as social media, application tracking systems, and even Artificial Intelligence (AI) and blockchain, to support talent recruitment. As many companies started to face recruiting challenges, they have incorporated e-hiring to their candidates' selection process. This type of e-recruiting has changed the corporate recruiting process thoroughly. However, there are still many companies struggling in their hiring process, unclear about what technology to use and how. Some of them hesitate about whether it is worthy of the investment in technical tools. For them, "to apply technology in recruitment or not" is still a question.

This research aims to provide some insights from the existing practices of applying technology in the recruitment process. Furthermore, this research is to find out how technical tools have changed the hiring process, and whether technology has benefited recruitment. By identifying the current trend of applying technology in recruitment, this research aims to help HR professionals determine if they should invest in e-recruitment and how to conduct erecruitment effectively. 
Specifically, this research asks the following research questions:

RQ1: What technological tools have been used in the recruitment process?

RQ2: How has technology software impacted the recruitment process?

RQ3: What the recruitment processes have benefited the most from technology?

RQ4: Are technology tools for recruitment easy to use?

RQ5: At what point would artificial intelligence impact recruiting?

\section{LITERATURE REVIEW}

Recruitment is a challenge for both job candidates and the recruiters. Finding a job is often the very first challenge for students after their graduation and sometimes still a life-changing event for experienced professionals. Recruitment is also a continuous problem for every company and organization if they want to maintain and sustain a strong team in the midst of market competitions and job turnovers. Technology could be one of the answers to address this problem. As the talent market becomes more competitive and global, it is important to study the technology use in recruitment and staffing practices. For employers wishing to have a competitive advantage in the labor market, it is a necessity to adopt the advanced technology in recruitment.

The use of technology in employee recruitment includes different practices and offers several advantages. Since popular recruitment technology in the market often incorporates both social media and cloud computing. This study focuses on the literature review on these two technologies specifically.

Social technologies have been used in many organizational functions and for multiple purposes. Social media can be used in human resource management (Tufts et. al., 2014; Wolf et. al., 2014) for recruitment and candidate selection, just like their applications in communications, sales and marketing. Platforms such as LinkedIn, Facebook and Twitter are used to post job advertisements, attract job applicants, and pre-screen applicants (Caers \& Castelyns, 2010). Carrillat et. al. (2014) show that with the use of social media, not only the employer brand and attractiveness have been improved, the recruitment process has also become more dynamic, relational, and authentic.

To make a better financial decision related to technology and recruitment, it is also critical to discover the benefits of the investment in e-recruitment process integration in comparison to separate e-recruitment investments. The study by In (2011) has shown that the optimal investment in the e-recruitment process integration results in a lower total cost than the separate e-recruiting investments. By integrating the e-recruitment process, it would be more efficient and cost saving. Cloud computing could offer one of the solutions to support the integration of erecruitment process.

\section{RESEARCH METHODOLOGY}

This research applies both quantitative and qualitative methods. In the quantitative data analysis, both primary and secondary datasets are used. The primary datasets were collected through a survey designed for this research. The survey targets human resource professionals who are involved in recruitment and focuses on HR professionals' views on the technology's impact on hiring. The survey has the following questions:

Q1: “What is the name of technology or software that is used the most in recruitment?” It is an open-ended question.

Q2. "How has technology software impacted the recruitment process?” and respondents are asked to rate on a fivepoint scale ranging from "technology worsens recruitment process significantly" to "technology improves recruitment process significantly.”

Q3. "In what step do you think has improved the most?" and respondents can select from "Sourcing candidates," "Screening candidates," "Scheduling interviews," "Engaging with candidates," "Interviewing Candidate," and "Onboarding."

Q4: "Is it easy for me to master the tools?" and respondents are asked to rate on a five-point scale ranging from "very hard to master" to "very easy to master." 
The secondary dataset is from the existing Harvey Nash survey 2018. The survey provided raw .csv data file for download. The question selected for this research was "At what point do you think artificial intelligence would impact recruiting workforce?" Respondents are asked to select one from "now, it is already happening," "in the next 2-5 years," "in the next 6-10 years," "in the next 11-20 years," "in more than 20 years," and "never."

In the qualitative part of the research, information was collected through interviews. HR from recruiting agency companies and recruiters from corporates for campus recruiting were interviewed. For these two types of firms, interviews were conducted with both manager and non-manager levels of recruiters. The interviews followed similar flow of the survey conducted, but in more details and with more probing questions to further understand the respondents' knowledge and perceived value of technology in the hiring process. The interviews covered questions about the software they were using for hiring, their experience of using the software, what role technology played in their day-to-day work, how technology affected the industry, and their thoughts about the future trends, and so on.

\section{RESULTS}

The data collection for the survey was conducted between February and April 2019. The survey was distributed with the help of two assistants. Altogether, 50 responses from HR professionals in four different companies in different industries were received. Their responses answered the first four research questions as follows:

\section{RQ1: What technological tools have been used in the recruitment process?}

Figure 1 shows that the recruiting teams use various technology for recruiting. Among the 50 respondents, most used software for recruiting are chosen to be Workday (24\%), SmartRecruiters (22\%), Taleo (18\%), iCIMS (16\%), Kenexa (10\%), and others (10\%).

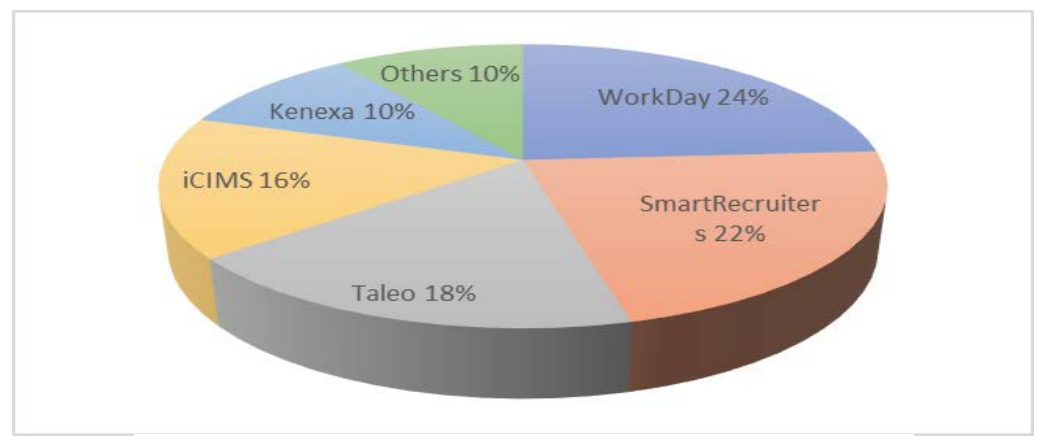

Figure 1. Most used technology/software for recruiting

\section{RQ2: How has technology software impacted the recruitment process?}

Figure 2 shows that out of all 50 participants, no one thinks that technology makes the recruiting process worse, and only $6 \%$ feels that nothing has changed. $94 \%$ of the respondents reported that technology has improved the recruit process: $54 \%$ of the respondents indicated that technology has improved the recruitment process significantly and $40 \%$ indicated the slight improvement. If "improve significantly" is considered as a score of 5 and "worsen significantly" as a score of 1 , then the average perceived technology improvement on recruiting process on a 1-5 scale is 4.48 out of 5 . Hence, it is perceived by the HR professionals that technology has a quite positive impact on the recruitment process. 


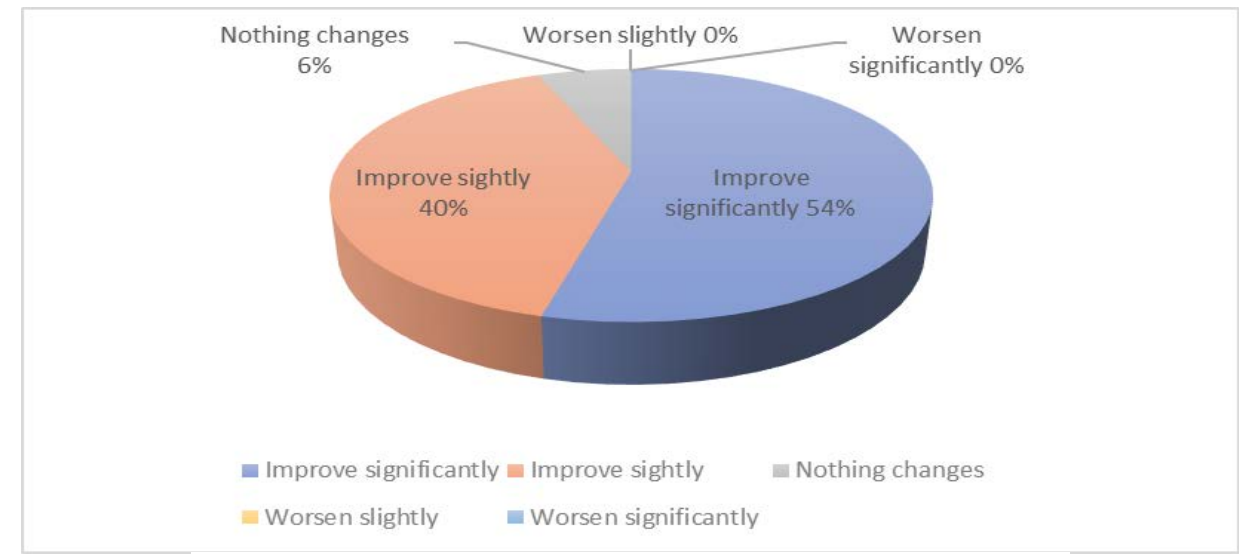

Figure 2. Perceived technology's impact on recruiting process

\section{RQ3: What the recruitment processes have benefited the most from technology?}

Figure 3 shows the number of respondents out of total 50 who rated the most improved stage among the six stages in the recruitment. Overall, it shows that technology improves the most at the earlier stages of sourcing candidates (28\%) and screening candidates (38\%), than at the later stages of scheduling interviews (10\%), interviewing candidates (6\%), engaging candidates (6\%), and onboarding (12\%). The percentage indicates the agreement from the percentage of the 50 survey respondents.

In what step do you think improve the most?

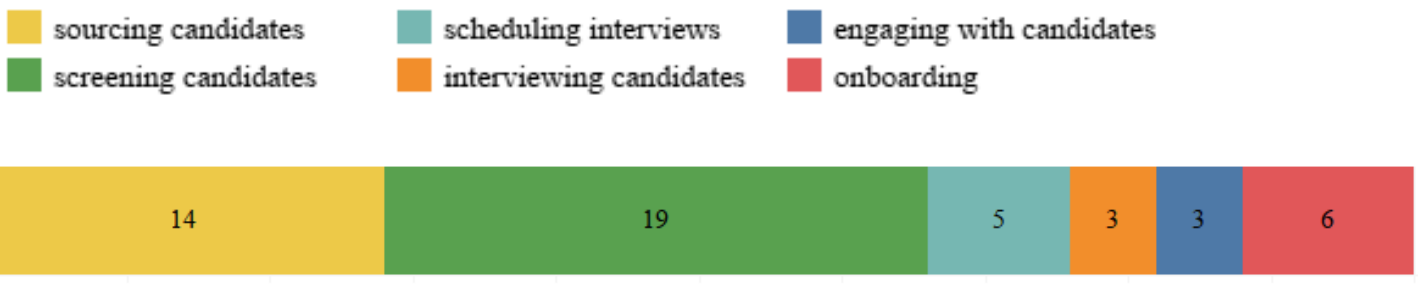

Figure 3. Technology's improvement at different stages of the recruitment process $(n=50)$

\section{RQ4: Are technology tools for recruitment easy to use?}

Figure 4 shows the perceived easiness to master the recruitment technology. 26\% of the respondents perceived "very easy,” 36\% perceived “easy,” 30\% perceived “normal,” 6\% perceived "hard,” and 2\% perceived "very hard.”

To aggregate the results, $58 \%$ percent of the HR professionals think that it is easy/very easy to use the tools, and only $8 \%$ feels that it is hard to manage the software they are using. Overall, if "very easy" is considered as a score of 5 and "very hard" as a score of 1 , then the average easiness on a 1-5 scale is 3.78 out of 5. 


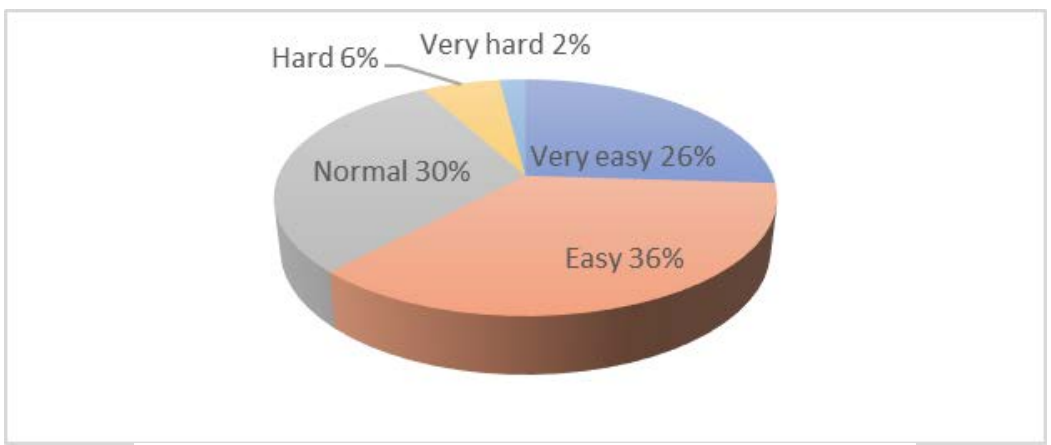

Figure 4. Easiness to master the recruitment technology

The qualitative interviews provide supplementary and rich contextual information to the survey questions. The interviews show that companies use the technology/software depicted in Figure 1 for hiring new employees and internal referral programs. These robust, cloud-based talent acquisition software are used from sourcing to hire. At the sourcing stage, these software platforms incorporate with the popular job posting sites, such as LinkedIn, Glassdoor and Indeed, to attract candidates for the roles and integrate all the information. Recruiters also use these software platforms in reviewing applications, communicating with candidates, and scheduling interviews. Large companies that have tremendous demand for contractors and consultants hiring usually have separate system or software to manage the process, such as SAP Fieldglass and ADP. They also involve third party staffing companies to facilitate the sourcing process.

The qualitative interviews also show the crucial positive changes that technology brings in. Large companies always need to recruit across different states/cities, and at experience levels, the software provides a very efficient way to unify the hiring process. Posting jobs and sometimes modifying the job description can be really redundant work especially when posting jobs at the different websites. The external job board synchronization is a very impressive function, which will synchronize the jobs posted at the different job portals and keep all of these postings synchronized and up to date.

After posting jobs, companies are often faced with the problems that too many people are interested in getting the role, and they are afraid that they would miss strong candidates if the waiting time increases. One of the interviewees emphasized that his/her company has completely removed the traditional manual resume screening, and this decision makes the screening cost plummet at least 60 percentage. The company can quickly identify and contact the top candidates, and the time to hire has been reduced significantly. The cloud-based platform makes it easier to manage candidates and interviews across the company. The recruiting team can revisit the previous candidates in the talent pool for similar roles. In addition, the seamless integration with different existing systems or social media has done a great job to improve the candidate flow. Along with the function of detecting duplicate candidates, there was no extra workload brought to recruiters for filtering applications. In addition, software nowadays can not only handle massive amount of resume data but also protect the data security and privacy at the same time.

The qualitative interviews also show that most of the recruiters state that tools are easy and straightforward even for people who just start their career in human resources. For most of the tool functions, there are usually instructions that can be easily referred to. For complex functions, step-by-step videos are available for most of the time. In additional, the FAQ section or help center are usually available to provide more information.

\section{RQ5: At what point would artificial intelligence impact recruiting?}

The Harvey Nash survey 2018 has 820 respondents with 127 different job titles from 38 countries. These 38 countries cover mainly North American, Europe, Australia, and Japan. This research specifically cares about the technology of Artificial Intelligence (AI). When discussing specifically about when AI changes the recruiting world, the Harvey Nash survey shows that out of 624 participants who answered this question, 163 (26\%) respondents believed that it is already happening, $46 \%$ believed that it would happen in the next 2 to 5 years, $20 \%$ believed that it would happen in the next 6-10 years (see Table 1 and Figure 5). In total, an overwhelming 92\% of the responses 
positively expect the AI change to come within the next 10 years. As AI such as machine learning has become popular in many industries, it makes sense that the recruiting process can also be changed and improved by such modern technology.

Table 1. Time expectation of artificial intelligence impacting recruitment (Harvey Nash Survey, 2018)

\begin{tabular}{|c|c|c|}
\hline $\begin{array}{l}\text { "At what point do you think artificial intelligence } \\
\text { would impact recruiting workforce?" }\end{array}$ & \# of respondents & $\%$ of respondents \\
\hline Now, it is already happenin & 163 & $26 \%$ \\
\hline In the next $2-5$ years & 288 & $46 \%$ \\
\hline In the next $6-10$ years & 124 & $20 \%$ \\
\hline In the next $11-20$ years & 28 & $4.5 \%$ \\
\hline In more than 20 years & 9 & $1.5 \%$ \\
\hline \multirow{2}{*}{$\begin{array}{l}\text { Never } \\
\text { Total }\end{array}$} & 12 & $2 \%$ \\
\hline & 624 & $100 \%$ \\
\hline
\end{tabular}

Figure 5. Time expectation of artificial intelligence impacting recruitment

\section{DISCUSSIONS}

This study shows that multiple software has been used for recruiting over the last few years, which generally covers different stages of recruiting process, from sourcing to hiring. Job posting sites attracts candidates and collects information at the sourcing stage; and HR reviews applications, communicates with candidates and schedules interviews at the hiring stage. Most of participants of the survey gave positive feedback on using technology in recruiting process. Key benefits of e-recruiting are time reduction and efficiency improvement. It is due to a large number of job openings posted and even more applications received. Applying technology into recruitment creates a unique platform to manage job posting and job application effectively. Both screening process and candidate selection become less time consuming. Even interview process gets much smoother than before. The technology makes the entire hiring process better integrated and better organized. HR can easily change and adjust the job postings and locate potential talents among hundreds if not thousands of job applicants.

Integrating technology into human resources is also not as complicated as expected. Software and other recruiting tools are very easy to use. Technical support is also available most of the time. According to the responses from the participants, e-recruiting has become much more popular than before and will be widely used in the next few years. Technology tools have positive impact on the recruiting process. It creates a manageable platform for job openings, attracts a lot more applicants through increased visibility on websites, collects and organizes hiring information effectively and efficiently, shortens the candidate screening and selection time, and helps to navigate ideal candidates with specific requirements. However, it also increases the total volume of job applications, which theoretically adds workload for human resource professionals. But due to overall improved effectiveness of the hiring process, the cumulative influence of e-recruiting still seems positive. In addition, it improves multiple stages of the staffing process. It changes the way of reviewing applications, communicating with candidates, scheduling interviews, and facilitating the sourcing process. More importantly, it even influences the overall structure of the recruiting market. Lots of labor markets in big cities, like New York City and San Francisco, start to shift towards 


\section{Issues in Information Systems \\ Volume 21, Issue 4, pp. 9-17, 2020}

getting tremendous demand for contractors and consultants. Therefore, third party staffing companies have also taken advantages of e-recruiting, which plays a significant role in current labor market.

Based on this study and the literature review, there is a lot of positive feedback from HR teams using technology tools in recruitment process. It is suggested that HR teams should conduct research on e-recruiting, find the most fit technology tools for the company, and keep learning how to effectively use these tools to improve the recruiting process. As e-recruiting has been developed tremendously over the last few years, it is also getting a little bit complicated. Several factors need to be considered when applying technology into recruiting, including but not limited to company size, complexity of job requirements, and timeline of recruiting process. HR team is required to understand their company's structure comprehensively, which helps them determine which stages of the recruiting process are on the top of their priority list. If there is a budget constraint, HR team should pay attention to what software will result in more improvement on the hiring process, or in what steps e-recruiting improves the most, or simply which is under the limit of budge.

Implementing these technology tools into recruiting also requires continuous study. Since these technology tools, including social media, have been developed so rapidly, it is necessary to keep up with the fast growth rate of technology. HR team should anticipate future changes in the labor market and get well prepared for emerging technology.

With appropriate setup and organized training program, HR teams could easily get started to implement technology tools into their day-to-day recruiting work. As discussed in the previous sections, positive impacts have been brought into recruiting process by utilizing technology. Sourcing candidates and screening candidates received the highest votes when improvement in different stages were discussed in the survey. Software helps recruiters save a great amount of time in both stages because sourcing candidate and screening candidates used to require manually sorting, arranging, analyzing, and cleaning up candidate information. These works will take a huge amount of time, which can be simply replaced by automatic data analysis performed by certain software. Posting jobs and changing job description can also be time-consuming work, especially when several jobs have been posted in different job sites. Technology, such as job board synchronization, could easily allow recruiters to keep all job postings up to date, and dramatically reduce the time of this type of repeated works.

Another major feature of e-recruiting is that recruiting software, like any other software utilizing big data, can handle massive amount of candidate information. The good news is that big data, at the same time, can also protect data security and user privacy. The advantages from big data for e-recruiting are still in need of exploring.

This research has shown the benefits of applying technology in recruiting process. There are limitations of this research as well. First, surveyed sample size can be larger. In addition, data collection should cover as many sample group types as possible. Diverse groups of participants can help reduce sampling bias. Since opinions from HR teams have been used to answer several research questions, it is critical to make sure that the participants come from different HR teams and in different industries. As personal experience and work atmosphere could have great impacts on surveyed results, larger sample sizes and more diverse groups of interviewees could improve the generalizability of this research.

This research can also be extended to additional questions such as examining the impact of company size on different recruiting technology tools and analyzing cost savings from e-recruiting quantitatively. With more variables in a bigger dataset, some statistic models, such as regression, can be used to test which variables have significant impact on cost saving per hire.

Another major limitation is the timeliness of this research. As information technology, especially social media and artificial intelligence, has been developed so rapidly over the last decade, applications of e-recruiting should be examined frequently. Staffing companies and HR teams will continue studying the current labor market and the growing trend of technology. It will always be a challenge for company to succeed and to grow; hence, recruiting process, which plays a significant role in company's development, should receive proper attention and investment. 


\begin{abstract}
SUMMARY
This research found that staffing and recruiting have had a revolutionary change in recent years. It has been observed of the structural change in labor market, the penetration of technology into recruiting, the impact of using social media on hiring process, and the benefits and the challenges of e-recruiting. In summary, applying technology into recruiting process has already brought and would continue bringing positive impacts on the labor market. Technology can simplify the screening process, reduce turnaround time, and improve the overall effectiveness for both job seeking and hiring. People looking for position openings can select the jobs that fit their lives, and by defining job titles, setting job types, giving salary ranges, etc. Companies are also capable of locating potential candidates by screening the basic requirements and questionnaires. All these features have become available when introducing technology tools into the recruiting process. The research results indicated that the application of erecruiting has positively improved the recruiting process and especially at the early stages of recruiting.
\end{abstract}

Because implementing technology into recruitment has become more popular than ever before, it is strongly recommended to get it started by those who have not adopted such technology. There should be cautions as well in adoption. Successful implementation requires research on different types of technology at the beginning, then HR teams need to pick the most appropriate software for the company. With limited budget or any other constraints, the selection of technology tools gets even more important. Focusing on development in sourcing stage and screening stage can be a good starting point. With the advanced development in AI, the sourcing and screening processes can get even more intelligent and automatic. There are great potentials for IT companies and staffing companies working together, and designing better integrated products and services.

For companies with more job postings, it is suggested to consider some features like external job board synchronization. This function allows quick and easy access to posting jobs and sometimes modifying the job description. It could dramatically reduce redundant workload and allow HR teams work on other tasks.

Large companies may also consider the data security and applicants' and employees' privacy with a higher priority. Software can handle data in a more effective and more secured way. At the same time, it potentially reduces the chance of information leakage and identity theft.

Cooperating with IT companies, recruiters will be able to use latest technology, receive timely technical support, providing useful feedback, and continuously learn and develop e-hiring tools. It requires closer collaboration and more integrated development process, but it has practical significance and broad market prospect.

Moreover, it has been proved that social media has significant influences on recruiting, and the entire social media market is getting bigger and more mature. It can be a very smart move to look into emerging social media platforms, innovative AI technology, and even blockchain. It will create a great vision of highly advanced e-recruiting process in the future.

\title{
REFERENCES
}

Bureau of Labor Statistics (U.S.) (2020) Job openings and labor turnover summary. Retrieved on March 11 ${ }^{\text {th }}, 2020$ at https://www.bls.gov/news.release/jolts.nr0.htm

Caers, R., Castelyns, V. (2010).LinkedIn and Facebook in Belgium: The influences and biases of social network sites in recruitment and selection procedures. Social Science Computer Review. 29(4).

Carrillat, F., d'Astous, A. \& Grégoire, E. (2014) Leveraging social media to enhance recruitment effectiveness: A Facebook experiment, Internet Research: Electronic Networking Applications and Policy, 24 (10).

Harvey Nash/KPMG CIO Survey. (2018) Retrieved from https://home.kpmg/xx/en/home/insights/2018/06/harvey-nash-kpmg-cio-survey-2018.html

Lee, I. (2011) Modeling the benefit of e-recruiting process integration, Decision Support Systems, 51(1): 230-239. 


\section{Issues in Information Systems}

Volume 21, Issue 4, pp. 9-17, 2020

Tufts, S., Jacobson, S. W. \& Stevens, M. (2014) Status update: Social media and local government human resource practices. Review of Public Personnel Administration, 35(2), 193-207.

Wolf, V., M., Sims, J. \& Yang, H. D. (2014). Social media utilization in human resource management. Web based communities and social Media 2014 conference. 